Katharina Rathberger, Sebastian Häusler*, Sven Wellmann, Marco Weigl, Florian Langhammer, Maria Victoria Bazzano, Andreas Ambrosch and Sara Fill Malfertheiner

\title{
SARS-CoV-2 in pregnancy and possible transfer of immunity: assessment of peripartal maternal and neonatal antibody levels and a longitudinal follow-up
}

https://doi.org/10.1515/jpm-2021-0166

Received April 6, 2021; accepted May 19, 2021;

published online June 14, 2021

\section{Abstract}

Objectives: In the current Severe Acute Respiratory Distress Coronavirus 2 (SARS-CoV-2) pandemic there is still great uncertainty about the effects of an infection in pregnancy especially regarding a possible fetal transmission of antibodies to SARS-CoV-2 and the longevity of this immunity.

Methods: Sixteen women who were infected with SARS-CoV-2 during pregnancy and their offspring were

Katharina Rathberger and Sebastian Häusler contributed equally to this work.

*Corresponding author: Sebastian Häusler, MD, University Department of Gynecology and Obstetrics, Hospital St. Hedwig of the Order of St. John, University Medical Center Regensburg, Steinmetzstr., 1-3, 93049 Regensburg, Germany; and Department of Obstetrics and Gynecology, School of Medicine, University of Würzburg, Josef-Schneider-Strasse 4, 97080 Würzburg, Germany, E-mail: sebastian.haeusler@barmherzige-regensburg.de Katharina Rathberger and Marco Weigl, University Department of Obstetrics and Gynecology at the Hospital St. Hedwig of the Order of St. John, University of Regensburg, Regensburg, Germany

Sven Wellmann and Florian Langhammer, Department of Neonatology, University Children's Hospital Regensburg (KUNO) at the Hospital St. Hedwig of the Order of St. John, University of Regensburg, Regensburg, Germany

Maria Victoria Bazzano, Laboratory of Translational Perinatology, University Department of Obstetrics and Gynecology at the Hospital St. Hedwig of the Order of St. John, University of Regensburg, Regensburg, Germany

Andreas Ambrosch, Institute of Laboratory Medicine, Microbiology and Hygiene, Hospital of the Order of St. John, Regensburg, Germany Sara Fill Malfertheiner, University Department of Obstetrics and Gynecology at the Hospital St. Hedwig of the Order of St. John, University of Regensburg, Regensburg, Germany; and WECARE Research and Development Campus Regensburg at the Hospital St. Hedwig of the Order of St. John, University of Regensburg, Regensburg, Germany included. The antibody response to SARS-CoV-2 was measured in mother and umbilical cord blood peripartum and in a follow-up examination 6-11 weeks after birth. Medical history, symptoms regarding SARS-CoV-2, obstetric and neonatal information were queried following recommendations by the WHO.

Results: A total of $73 \%$ of the women and one third of the infants developed antibodies to SARS-CoV-2 spike (S) protein receptor binding domain (RBD), with a long interval between infection and birth proving favorable for a transplacentar transfer of antibodies to the neonates. All infants showed declining or vanishing antibody-titers in the follow-up examination, while the titers of their mothers were stable or even increased.

Conclusions: Our results demonstrate that transplacental transfer of SARS-CoV-2-specific antibodies is possible, but also indicate that the immunity that may be gained as a result might decrease in newborns postpartum. This provides important evidence that could be useful for further studies covering vaccination during pregnancy.

Keywords: IgA; IgG; immunity; peripartum period; pregnancy; SARS-CoV-2; screening.

\section{Introduction}

In the end of 2019 the Severe Acute Respiratory Distress Coronavirus 2 (SARS-CoV-2) emerged in China. Soon the virus and the associated illness known as COVID-19 (Coronavirus disease 2019) became a global pandemic [1]. The rapid spread raised concerns over a potentially vulnerable group: pregnant women. Due to physiological changes during pregnancy, which make them more susceptible for complications of respiratory infections, pregnant women were thought to be at a higher risk for adverse outcomes of COVID-19 [2, 3]. Other coronavirus diseases like MERS-CoV (Middle East Respiratory Syndrome Coronavirus) and SARS-CoV-1 showed significantly more severe courses in this group [4]. Fortunately, the 
largest proportion of pregnant women infected with SARS-CoV-2 showed asymptomatic or mild courses. However, there are individual case reports of severe courses of COVID-19 in pregnancy [3, 5, 6]. Furthermore, current data shows higher rates of intensive care unit (ICU) admissions, invasive ventilation and death in pregnant woman compared with nonpregnant women with COVID-19 [32]. Another concern raised was the risk an infection could pose to the unborn child. If the vertical transmission of the virus is possible and which consequences it would have is still not fully clarified but the risk seems to be relatively small $[2,7]$. But even with the ground breaking research performed in the last months, the long-term consequences of an infection with SARS-CoV-2 during pregnancy for mother and child including a possible transfer of immunity stay mostly unknown. With this study the maternal and neonatal outcome and antibody response after a SARS-CoV-2 infection during pregnancy will be explored.

\section{Materials and methods}

The study was designed as a prospective longitudinal study focusing on the immune response to SARS-CoV-2 in mother and child after an infection with the virus during pregnancy. It includes all patients who presented with a SARS-CoV-2-infection during pregnancy at the university maternal hospital St. Hedwig in Regensburg, Germany from April to December 2020. A reverse transcription polymerase chain reaction (RT-PCR) and/or antibody testing in maternal serum was performed in every patient admitted to the hospital as an admission screening to detect an ongoing or former infection with SARS-CoV 2 [8]. RT-PCR was performed according to the protocol established by Drosten et al. [9]. Additionally, the antibody response in umbilical cord blood was measured. Symptoms and risk factors of SARS-CoV-2 were queried with a standardized questionnaire following recommendations issued by the World Health Organization in order to standardize research in SARS-Cov2-infections [10, 11]. Medical history, obstetrical and neonatal information was available. In postpartum follow-up-examinations six to twelve weeks after delivery maternal, paternal and child serum and breast milk was tested for antibody response to SARS-CoV-2. RT-PCR was performed in mother, father and child and they were screened for residual symptoms. Figure 1 gives an overview of the chronological course of the study.

The detection of antibodies was performed via Elecsys ${ }^{\circledR}$ AntiSARS-CoV-2 S RBD assay (Roche, Switzerland) which tests for antibodies to the SARS-CoV-2 spike (S) protein receptor binding domain (RBD). The assay was performed according to the manufacturer's protocol. It detects predominantly immunglobulin (Ig)G, but also includes IgA and IgM [12]. A measured value above $0.8 \mathrm{U} / \mathrm{mL}$ is considered positive. The breast milk samples were centrifuged and the supernatant was used for the assay. In five neonatal and four paternal samples IgG to SARS-CoV-2 was detected instead using IgG enzymelinked immunosorbent assay (ELISA) from EUROIMMUN AG (Lübeck, Germany). The cut-off value for a positive result in IgG is above $1.2 \mathrm{U} / \mathrm{mL}$. The study has been approved by the Ethics Committee of the University of Regensburg (reference number: 20-1828-101). All participating parents provided written informed consent.

The data handling and evaluation were performed using Microsoft Excel 2019 (Microsoft, USA). Due to the low number of study participants statistical evaluation was omitted.

\section{Results}

In total 16 women and their children were included in the study. All women were tested positive for SARS-CoV-2 during pregnancy with none of them infected with a variant of concern (VOC) such as B.1.1.7 or B.1.351. One patient was excluded because the suspected SARS-CoV-2 infection was not confirmed by RT-PCR. $27 \%$ of the patients were overweight (body mass index [BMI] 25.0 to <30) and 21\% were

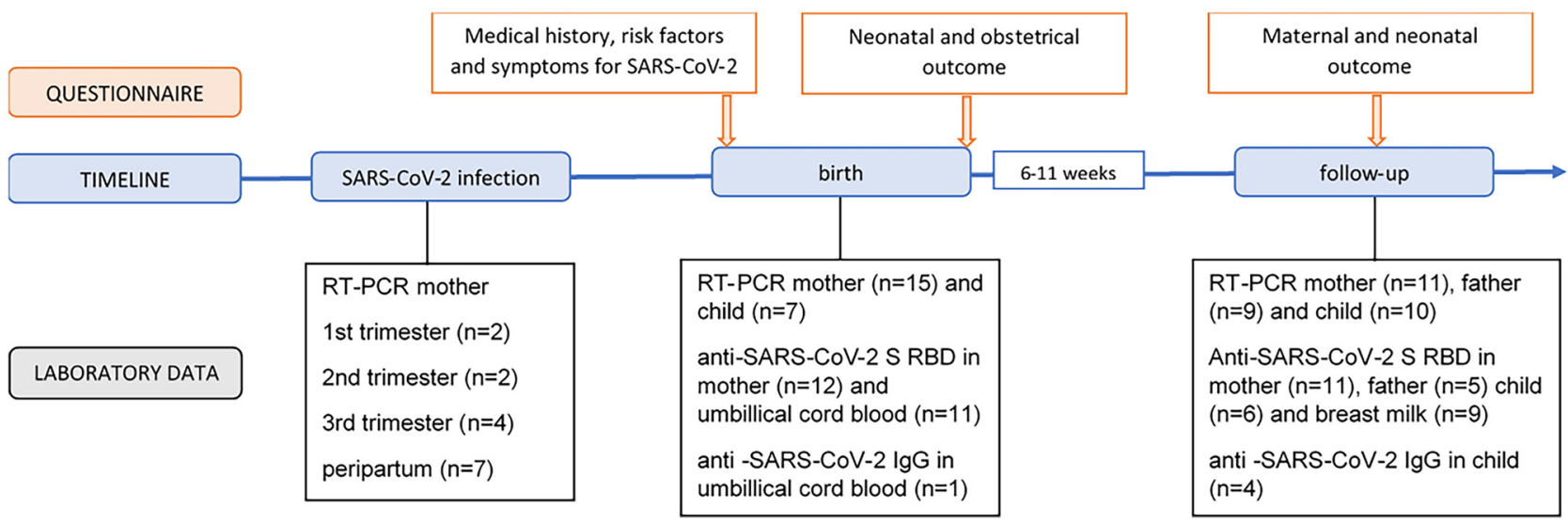

Figure 1: Flow of participants throughout the study period including times of collection of questionnaire and laboratory data. The SARS-CoV-2 infection during pregnancy was confirmed via RT-PCR. Peripartum antibody levels to SARS-CoV-2 were determined in mother and child followed by a follow-up examination of mother, father and child 6-11 weeks after delivery. In total three questionnaires queried medical history, relevant risk factors and symptoms for COVID-19, peripartal management and the outcome of mother and child. 
obese (BMI 30.0 or higher) before pregnancy. Apart from that none of them had severe pre-existing conditions, especially no cardiopulmonary diseases. Regarding pregnancy complications, two of the women were diagnosed with preeclampsia, one associated with intrauterine growth retardation (IUGR). Eight were infected with SARS-CoV-2 prepartum and seven peripartum (maximum 14 days before birth, Figure 1 and Table 1). Among the prepartum infections two were in the first and in the second trimester respectively and four took place in the third trimester (Table 1). For all patients the origin of infection could not be precisely determined. Two just returned from travel abroad and eight hat close relatives who tested positive for SARS-CoV-2. The majority of the women had mild courses of COVID-19, mild dyspnea and headache being the most common reported symptom
(27\%) followed by anosmia and rhinorrhea (20\%). Other symptoms included cough, arthralgia, myalgia, fatigue and conjunctivitis. Over half of the participants were completely asymptomatic. In one case (Patient ID 14 in Table 1) the woman presented at GW (gestational weeks) $30+2$ with severe dyspnea and was tested SARS-CoV-2 positive. Because of the deteriorating general condition with repeated drops in oxygen saturation a cesarean section (c-section) was performed at GW $30+2$. Following the surgery, the patient was admitted to intermediate care unit. Oxygen supplementation but no invasive or noninvasive ventilation was applied. The patient could be transferred to the general ward after three days and was released ten days after the c-section. She showed no residual symptoms in the postpartum follow-up. In the cohort seven patients delivered via c-section, six via

Table 1: Antibody levels peri- and postpartum in mother, father and child. Values labeled "IgG" (Immunglobuline G) were measured using EUROIMMUN ELISA. Colour coding for gestational age: green for first, blue for second, yellow for third trimester at time of SARS-CoV-2 infection; red for peripartum infection. Elevated values are marked in bold letters. "/" means not done. GW, gestational weeks.

\begin{tabular}{|c|c|c|c|c|c|c|c|}
\hline \multirow[t]{3}{*}{$\begin{array}{l}\text { Patient } \\
\text { ID }\end{array}$} & \multirow{3}{*}{$\begin{array}{l}\text { GW } \\
\text { when } \\
\text { testing } \\
\text { positive } \\
\text { for SARS- } \\
\text { CoV-2 }\end{array}$} & \multirow{3}{*}{$\begin{array}{ll}\text { GW } & \text { at } \\
\text { time of } \\
\text { birth }\end{array}$} & \multicolumn{5}{|c|}{$\begin{array}{l}\text { Anti-SARS-CoV S, U/mL } \\
\text { IgG, U/mL (EUROIMMUN ELISA) }\end{array}$} \\
\hline & & & \multirow{2}{*}{$\begin{array}{l}\text { Peripartum } \\
\text { mother }\end{array}$} & \multirow{2}{*}{$\begin{array}{l}\text { Umbillical } \\
\text { cord blood }\end{array}$} & \multirow[t]{2}{*}{ Breast milk } & \multicolumn{2}{|c|}{ Postpartum control } \\
\hline & & & & & & Mother & Child \\
\hline \multicolumn{8}{|c|}{ SARS-CoV-2 -infection in first trimester } \\
\hline 1 & 3 & 39 & 61.6 & 102.3 & / & 78.21 & 31.49 \\
\hline 2 & 6 & 37 & $<0.40$ & / & / & / & / \\
\hline \multicolumn{8}{|c|}{ SARS-CoV-2 -infection in second trimester } \\
\hline 3 & 21 & 41 & $>250$ & $>250$ & 1.03 & $>250$ & 99.06 \\
\hline 4 & 15 & 42 & $>250$ & / & 2.11 & / & / \\
\hline \multicolumn{8}{|c|}{ SARS-CoV-2 -infection in third trimester } \\
\hline 5 & 36 & 38 & 44.63 & 5.17 & 1.41 & $>250$ & IgG 0.5 \\
\hline 6 & 29 & 37 & $<0.40$ & $<0.40$ & $<0.40$ & $<0.40$ & l \\
\hline 7 & 31 & 40 & 2.86 & 1.53 & $<0.40$ & 8.48 & $\lg G 0.1$ \\
\hline 8 & 37 & 39 & 21.38 & $<0.40$ & 0.72 & 21.46 & $<0.40$ \\
\hline \multicolumn{8}{|c|}{ SARS-CoV-2 -infection in third trimester - peripartum (14 days before birth to birth) } \\
\hline 9 & 39 & 40 & $<0.40$ & $<0.40$ & / & 6.45 & $\operatorname{lgG} 0.1$ \\
\hline 10 & 41 & 41 & $<0.40$ & $<0.40$ & / & / & I \\
\hline 11 & 39 & 39 & 93.90 & IgG 9.0 & 3.30 & $>250$ & IgG 1.4 \\
\hline 12 & 37 & 39 & I & $<0.40$ & $<0.40$ & 16.19 & $<0.40$ \\
\hline 13 & 41 & 41 & $<0.40$ & $<0.40$ & I & I & / \\
\hline 14 & 31 & 31 & 12.3 & $<0.40$ & / & 204 & $<0.40$ \\
\hline 15 & 40 & 40 & / & / & $<0.40$ & 9.65 & $<0.40$ \\
\hline
\end{tabular}




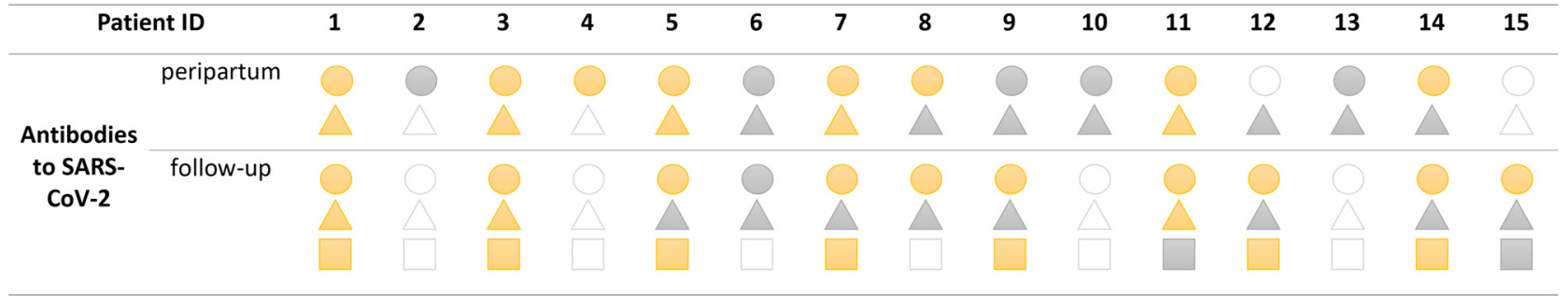

Figure 2: Antibody levels to SARS-CoV-2 in all participating families peripartum and in the postpartum follow-up. The majority of mothers developed antibodies at the time of follow-up while the antibody status of the infant seemed to depend on the height of the maternal antibody level at birth and the time between birth and the infection with SARS-CoV-2. Most of the fathers were tested positive for antibodies to SARS-CoV-2 (Circle [mother], triangle [infant], father [rectangle], positive: Yellow, negative: Grey, not done: clear).

spontaneous and two via instrumental vaginal delivery. Except from case 14 the obstetric management was not affected by the SARS-CoV-2-infection. In addition to case 14 , there were two other preterm births, both in the 37th week of gestation. The APGAR scores of all children were adequate, only the aforementioned child delivered at GW $30+2$ had and 1 min Apgar of 6 . The umbilical cord arterial $\mathrm{pH}$ was above 7.10 in all neonates. Three children required admission to the neonatal unit. Causes of admission were preterm birth and/or hyperbilirubinaemia. None of the neonates were tested positive for SARS-CoV-2 in RT-PCR. Eleven women took part in the follow-up at the end of their puerperium, ten of them were accompanied by their child. All of them reported no residual symptoms of COVID-19 and the children showed normal development in the medical check-ups. The detected antibody levels against SARS-CoV-2S RBD can be found in Table 1 and an overview of all participating families is shown in Figure 2. Peripartum eight women had elevated antibody levels against SARS-CoV-2 S RBD. In four of these women SARS-CoV-2 S antibodies could be detected in the breast milk $(\mathrm{n}=9)$. In the umbilical cord blood of five neonates elevated antibody levels could be detected ( $n=12)$, all born to antibodypositive mothers (Figure 3 ).

The elevated antibody levels persisted in the postpartum follow-up in all mothers and some of the infants but whereas the maternal antibody load was stable or increased (Figure 4), the children showed a lower antibody response to SARS-CoV-2 S RBD in the follow-up examination compared to the measurements in umbilical cord blood.

In two cases (5 and 7) in which the infection had occurred in the third trimester, no elevated antibody titers could be detected in the follow-up examination of the infant.

In half of the women who were infected with SARS-CoV-2 peripartum no antibodies against SARS-CoV-2S RBD could be detected neither in maternal nor in umbilical cord blood but all women except for one (case 6) showed a positive antibody response in the follow-up examination. However, the children of those mothers showed no antibody response to

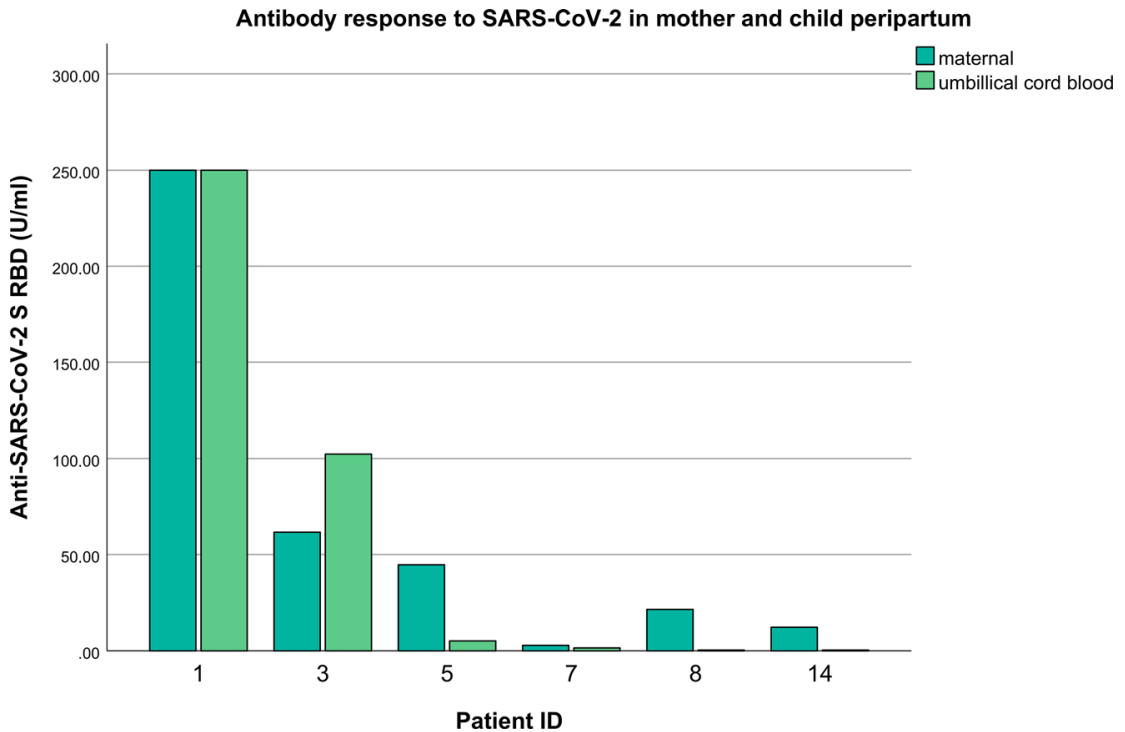

Figure 3: SARS-CoV-2 S RBD antibodies peripartum in mother and child. In this chart, only those cases are shown in which the women showed a positive antibody response peripartum and measurements from mother and child were available. All but one infant showed lower or the same antibody levels in umbilical cord blood compared to their mothers. 


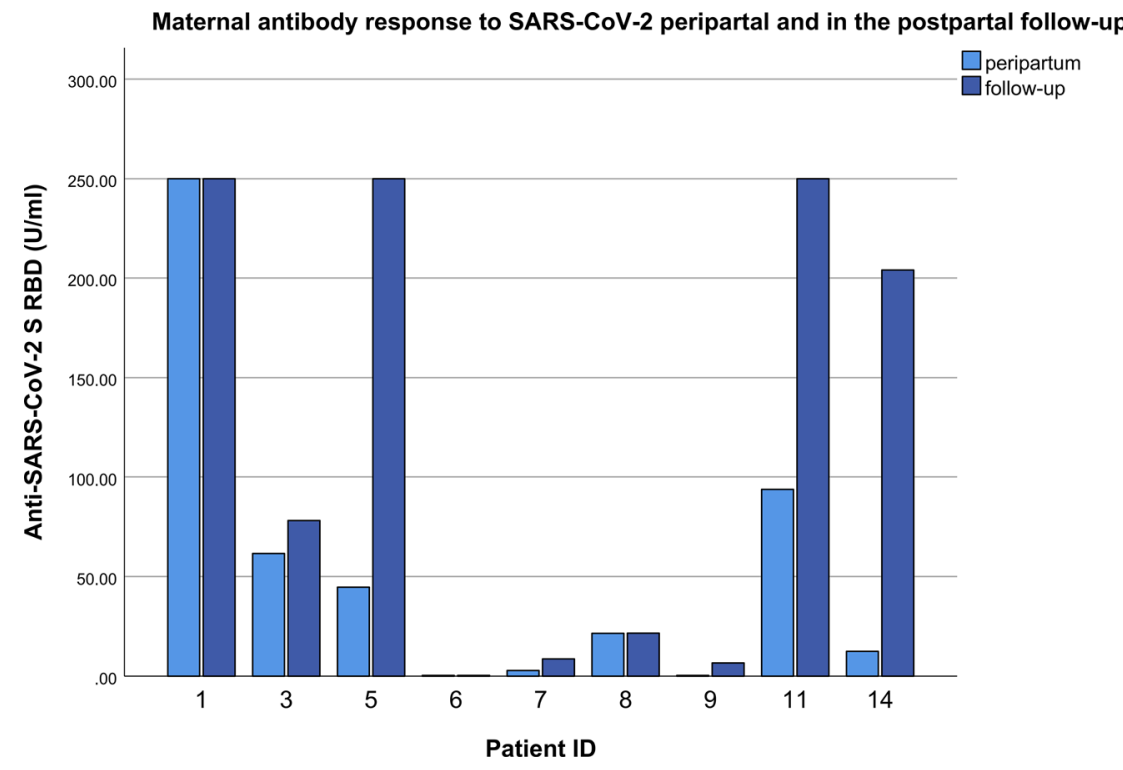

Figure 4: SARS-CoV-2 S RBD antibodies in maternal blood peripartal and at the end of puerperium.

All women showed the same or higher antibody levels in the follow-up compared to the antibodies to SARS-CoV-2 detected peripartum. In this chart only the cases with follow-up data are shown.
SARS-CoV-2 S RBD in the postpartum follow-up despite rooming-in with their mothers. $78 \%$ of the fathers were tested positive for SARS-CoV-2-specific antibodies $(\mathrm{n}=9)$.

\section{Discussion}

Pregnant women and neonates are a group particularly susceptible to complications of respiratory infections $[6,13$, 14]. Therefore, during the current pandemic it is important to explore the mechanisms of immunity in this vulnerable group. In this study of 15 women who were tested SARS-CoV-2 positive during pregnancy all but one patient showed mild to asymptomatic courses of COVID-19 and reported no residual symptoms in the postpartum followup. In the literature the majority of pregnant women had mild to asymptomatic courses [2, 3, 7, 15]. With regard to the neonatal outcome several studies suggest a higher prevalence of premature birth, low birth weight and c-section compared to SARS-CoV-2 negative mothers [3, 6 , $15,16]$. Four out of seven women who were tested positive for SARS-CoV-2 within 14 days before birth delivered by c-section and there were three preterm births in our study. However, only one c-section before term was due to a severe course of COVID-19. Apgar scores in newborns of SARS-CoV-2-positive mothers appear to be mostly adequate, which is also confirmed by our study $[15,17]$.

In our study, the majority of mothers who tested positive for antibodies at the time of delivery had been infected with SARS-CoV-2 for more than 14 days before giving birth. And those who had not yet developed an antibody response to SARS-CoV-2 at birth, developed a positive antibody-response in the follow-up at the end of puerperium except for one women who remained negative. This fits well with an average seroconversion time for SARS-CoV-2 specific IgG of 12-14 days after symptom onset and a peak at around three to seven weeks post symptom onset $[18,19]$. It has also been shown that some patients tested positive for SARS-CoV-2 in RT-PCR remain negative regarding specific antibodies, which could explain the seronegative results for SARS-CoV-2S RBD for cases 2 (data only up to delivery), and $6[18,20]$.

Vertical transmission could not be detected in any case, but our study results suggest a transplacental transmission of antibodies that might favor transient immunity in the newborn. A high maternal antibody titer and a long time period between maternal infection and birth seem to favor the transfer of antibodies to the fetus. Those findings align with current literature [21-24]. In addition to diaplacental transfer of specific antibodies we noted a robust transfer via breastmilk in those mothers with high titers at birth, which is in line with recent data [25, 26] and supports the recommendations to continue breastfeeding during mild-to-moderate maternal COVID-19 disease.

We also see some evidence that transplacental transfer of maternal antibodies to SARS-CoV-2 appears to be impaired. The antibody levels in cord blood were lower than in maternal blood in all but one case. This is in contrast to the fact that the maternal-to-cord ratio in IgG antibodies is known to be close to or above 1.0 [27].

Edlow et al. also found an inefficient transfer of maternal antibodies to SARS-CoV-2, particularly in third 
trimester infections [14, 22]. On the other hand, Flannery et al. [23] found the cord-to-maternal transfer ratio of antibodies to SARS-CoV-2 to be adequate.

More than three quarters of the fathers showed positive antibody levels to SARS-CoV-2. However, Madewell et al. [28] estimated the secondary transmission rate in households and families for this infection at about $16.6 \%$. The lower transmission rate could be justified by the closer contact between partners compared to household members, though another study found a 37\% risk for paturients to be antibody-positive if their partner tested seropositive for SARS-CoV-2 [29].

All children showed a decline in antibodies to SARS-CoV-2 S RBD in the follow-up examination. Moreover, two infants born to mothers infected with SARS-CoV-2 in the third trimester tested antibody-negative in the follow-up. This is consistent with findings from Gao et al. [30] who found decreasing SARS-CoV-2-specific IgG-levels in 11 neonates born to seropositive mothers. The possible transplacental transfer of antibody protection and its longevity is particularly relevant with regard to a possible vaccination of pregnant women against SARS-CoV-2. A recently published single case study describes a positive antibody titer to SARS-CoV-2 in umbillical cord blood after vaccination of the mother at 32 weeks of gestation [31]. However, our results indicate that vaccination against SARS-CoV-2 (as soon as it is deemed safe in pregnancy) might be advisable to be administered in the first or second trimester to allow for possible transmission of immunity to the child. To further validate our findings and gain even greater insight into SARS-CoV-2 infections in pregnancy and, in particular, the transmission and longevity of immunity to the newborn, further studies with higher case numbers are needed.

Acknowledgments: We are grateful to all obstetrics patient who participated in the study and to all staff members of our hospital supporting this research project. Moreover, we would like to thank Andrea Seitz for supporting the collection and management of data for this study.

Research funding: None declared.

Author contributions: Katharina Rathberger: Investigation (equal); Formal analysis (lead); Writing-review original draft (lead). Sebastian Häusler: Conceptualization (equal); Project administration (supporting); Resources (supporting); Supervision (equal); Writing-review original draft (supporting); Methodology (equal). Sven Wellmann: Investigation (equal), Conceptualization (supporting)r. Marco Weigl:
Investigation (equal); Methodology (supporting). Florian Langhammer: Investigation (equal). Maria Victoria Bazzano: Investigation (equal). Andreas Ambrosch: Investigation (supporting); Methodology (supporting); Validation (supporting). Sara Fill Malfertheiner: Conceptualization (equal); Investigation (lead); Formal analysis (supporting); Methodology (equal); Supervision (equal); Project administration (equal); Writing-original draft (supporting). All authors have accepted responsibility for the entire content of this manuscript and approved its submission.

Competing interests: Authors state no conflict of interest. Informed consent: Informed consent was obtained from all individuals included in this study.

Ethical approval: The study has been approved by the Ethics Committee of the University of Regensburg (reference number: 20-1828-101).

Availability of data and materials: The datasets used and/ or analyzed for this paper are available from the corresponding author on reasonable request.

\section{References}

1. Yan Y, Shin W, Pang YX, Meng Y, Lai J, You C, et al. The first 75 days of novel coronavirus (SARS-CoV-2) outbreak: recent advances, prevention, and treatment. Int J Environ Res Publ Health 2020;17: 2323-45.

2. Narang K, Enninga EAL, Gunaratne MDSK, Ibirogba ER, Trad ATA, Elrefaei A, et al. SARS-CoV-2 infection and COVID-19 during pregnancy: a multidisciplinary review. Mayo Clin Proc 2020;95: 1750-65.

3. Salem D, Katranji F, Bakdash T. COVID-19 infection in pregnant women: review of maternal and fetal outcomes. Int I Gynaecol Obstet 2020;152:291-8.

4. Pettirosso E, Giles M, Cole S, Rees M. COVID-19 and pregnancy: a review of clinical characteristics, obstetric outcomes and vertical transmission. Aust N Z J Obstet Gynaecol 2020;60: 640-59.

5. Ayed A, Embaireeg A, Benawadh A, Al-Fouzan W, Hammoud M, Al-Hathal $M$, et al. Maternal and perinatal characteristics and outcomes of pregnancies complicated with COVID-19 in Kuwait. BMC Pregnancy Childbirth 2020;20:754.

6. Barrero-Castillero A, Beam KS, Bernardini LB, Ramos EGC, Davenport PE, Duncan AR, et al. COVID-19: neonatal-perinatal perspectives. J Perinatol 2020;41:940-51.

7. Alzamora MC, Paredes T, Caceres D, Webb CM, Valdez LM, La Rosa $M$. Severe COVID-19 during pregnancy and possible vertical transmission. Am J Perinatol 2020;37:861-5.

8. Häusler S, Weigl M, Ambrosch A, Gruber R, Seelbach-Göbel B, Fill MS. Peripartal anti-SARS-CoV-2-lgA/IgG in asymptomatic pregnant women during regional SARS-CoV-2-outbreak [Internet]. J Perinat Med 2021;49:709-16. 
9. Corman VM, Landt O, Kaiser M, Molenkamp R, Meijer A, Chu DK, et al. Detection of 2019 novel coronavirus (2019-nCoV) by realtime RT-PCR. Euro Surveill 2020;25:23-30.

10. ISARIC. Coronavirus (COVID-19) case report form for pregnant women [cited 2021 Mar 23]. Available from: https://isaric.org/ wp-content/uploads/2021/02/ISARIC-COVID-19-CORECRF-02FEB21.pdf.

11. ISARIC. COVID-19 Core Case Report Form: acute respiratory infection clinical characterisation data tool [cited 2021 Mar 23].

12. Egger M, Bundschuh C, Wiesinger K, Gabriel C, Clodi M, Mueller T, et al. Comparison of the Elecsys ${ }^{\circledR}$ Anti-SARS-CoV-2 immunoassay with the EDITM enzyme linked immunosorbent assays for the detection of SARS-CoV-2 antibodies in human plasma. Clin Chim Acta 2020;509:18-21.

13. Rasmussen SA, Jamieson DJ, Uyeki TM. Effects of influenza on pregnant women and infants. Am J Obstet Gynecol 2012;207:S3-8.

14. Atyeo C, Pullen KM, Bordt EA, Fischinger S, Burke J, Michell A, et al. Compromised SARS-CoV-2-specific placental antibody transfer. Cell 2021;184:628-42.e10.

15. Yee J, Kim W, Han JM, Yoon HY, Lee N, Lee KE, et al. Clinical manifestations and perinatal outcomes of pregnant women with COVID-19: a systematic review and meta-analysis. Sci Rep 2020; 10:1-7.

16. Cavalcante MB, Cavalcante CTMB, Sarno M, Barini R, Kwak-Kim J. Maternal immune responses and obstetrical outcomes of pregnant women with COVID-19 and possible health risks of offspring. J Reprod Immunol 2021;143:1-10.

17. Chi J, Gong W, Gao Q. Clinical characteristics and outcomes of pregnant women with COVID-19 and the risk of vertical transmission: a systematic review. Arch Gynecol Obstet 2021; 303:337-45.

18. Post N, Eddy D, Huntley C, van Schalkwyk MCl, Shrotri M, Leeman $D$, et al. Antibody response to SARS-CoV-2 infection in humans: a systematic review. PLoS One 2020;15:e0244126.

19. Cosma S, Carosso AR, Corcione S, Cusato J, Borella F, Antonucci $M$, et al. Longitudinal analysis of antibody response following SARS-CoV-2 infection in pregnancy: from the first trimester to delivery. J Reprod Immunol 2021;144:1-3.

20. Röltgen K, Powell AE, Wirz OF, Stevens BA, Hogan CA, Najeeb J, et al. Defining the features and duration of antibody responses to SARS-CoV-2 infection associated with disease severity and outcome. Sci Immunol 2020;5:abe0240.

21. Preßler J, Fill Malfertheiner S, Kabesch M, Buntrock-Döpke H, Häusler S, Ambrosch A, et al. Postnatal SARS-CoV-2 infection and immunological reaction: a prospective family cohort study. Pediatr Allergy Immunol 2020;31:864-7.
22. Edlow AG, Li JZ, Collier A-RY, Atyeo C, James KE, Boatin AA, et al. Assessment of maternal and neonatal SARS-CoV-2 viral load, transplacental antibody transfer, and placental pathology in pregnancies during the COVID-19 pandemic. JAMA Netw Open 2020;3:e2030455.

23. Flannery DD, Gouma S, Dhudasia MB, Mukhopadhyay S, Pfeifer MR, Woodford EC, et al. Assessment of maternal and neonatal cord blood SARS-CoV-2 antibodies and placental transfer ratios. JAMA Pediatr 2021;e210038. https://doi.org/10. 1001/jamapediatrics.2021.0038.

24. Egerup P, Fich Olsen L, Christiansen A-MH, Westergaard D, Severinsen ER, Hviid KVR, et al. Severe Acute Respiratory Syndrome Coronavirus 2 (SARS-CoV-2) antibodies at delivery in women, partners, and newborns. Obstet Gynecol 2021;137: 49-55.

25. Demers-Mathieu V, Do DM, Mathijssen GB, Sela DA, Seppo A, Järvinen KM, et al. Difference in levels of SARS-CoV-2 S1 and S2 subunits- and nucleocapsid protein-reactive SIgM/IgM, IgG and SIgA/IgA antibodies in human milk. J Perinatol 2020;41: 850-9.

26. Pace RM, Williams JE, Järvinen KM, Belfort MB, Pace CDW, Lackey KA, et al. Characterization of SARS-CoV-2 RNA, antibodies, and neutralizing capacity in milk produced by women with COVID-19. mBio 2021;12:e03192-20.

27. Clements T, Rice TF, Vamvakas G, Barnett S, Barnes M, Donaldson $B$, et al. Update on transplacental transfer of IgG subclasses: impact of maternal and fetal factors. Front Immunol 2020;11: 1920.

28. Madewell ZJ, Yang Y, Longini IM, Halloran ME, Dean NE. Household transmission of SARS-CoV-2: a systematic review and meta-analysis. JAMA Netw Open 2020;3:e2031756.

29. Egerup P, Olsen LF, Christiansen A-MH, Westergaard D, Severinsen ER, Hviid KVR, et al. Impact of SARS-CoV-2 antibodies at delivery in women, partners and newborns; 2020.

30. Gao J, Li W, Hu X, Wei Y, Wu J, Luo X, et al. Disappearance of SARS-CoV-2 antibodies in infants born to women with COVID-19, Wuhan, China. Emerg Infect Dis 2020;26:2491-4.

31. Gill L, Jones CW. Severe Acute respiratory Syndrome coronavirus 2 (SARS-CoV-2) antibodies in neonatal cord blood after vaccination in pregnancy. Obstet Gynecol 2021;137:894-6.

32. Zambrano LD, Ellington S, Strid P, Galang RR, Oduyebo T, van Tong $T$, et al. Update: characteristics of symptomatic women of reproductive age with laboratory-confirmed SARS-CoV-2 infection by pregnancy status - United States, January 22-October 3, 2020. MMWR Morb Mortal Wkly Rep 2020;69:1641-7. 